\title{
Solar neutrino detection sensitivity in DARWIN via electron scattering
}

\author{
DARWIN Collaboration
}

J. Aalbers ${ }^{1}$, F. Agostini' ${ }^{2}$, S. E. M. Ahmed Maouloud ${ }^{3}$, M. Alfonsi ${ }^{4}$, L. Althueser ${ }^{5}$, F. D. Amaro ${ }^{6}$, J. Angevaare ${ }^{7}$, V. C. Antochi ${ }^{1}$, B. Antunovic ${ }^{8}$, E. Aprile ${ }^{9}$, L. Arazi ${ }^{10}$, F. Arneodo ${ }^{11}$, M. Balzer ${ }^{12}$, L. Baudis ${ }^{13}$, D. Baur ${ }^{14}$, M. L. Benabderrahmane ${ }^{11}$, Y. Biondi ${ }^{13}$, A. Bismark ${ }^{13,14}$, C. Bourgeois ${ }^{15}$, A. Breskin ${ }^{10}$, P. A. Breur ${ }^{7}$, A. Brown ${ }^{13}$, E. Brown ${ }^{16}$, S. Brünner ${ }^{7}$, G. Bruno ${ }^{11}$, R. Budnik ${ }^{10}$, C. Capelli ${ }^{13}$, J. Cardoso ${ }^{6}$, D. Cichon ${ }^{17}$, M. Clark ${ }^{18}$, A. P. Colijn ${ }^{7, b}$, J. Conrad ${ }^{1, c}$, J. J. Cuenca-García ${ }^{19}$, J. P. Cussonneau ${ }^{20}$, M. P. Decowski ${ }^{7}$, A. Depoian ${ }^{18}$, J. Dierle ${ }^{14}$, P. Di Gangi ${ }^{2}$, A. Di Giovanni ${ }^{11}$, S. Diglio ${ }^{20}$, D. Douillet ${ }^{15}$, G. Drexlin ${ }^{21}$, K. Eitel ${ }^{19}$, R. Engel ${ }^{19}$, E. Erdal ${ }^{10}$, A. D. Ferella ${ }^{22,23}$, H. Fischer ${ }^{14}$, P. Fischer ${ }^{24}$, W. Fulgione ${ }^{25}$, P. Gaemers ${ }^{7}$, M. Galloway ${ }^{13}$, F. Gao ${ }^{9}$, D. Giovagnoli ${ }^{20}$, F. Girard ${ }^{13}$, R. Glade-Beucke ${ }^{14}$, F. Glück ${ }^{19}$, L. Grandi ${ }^{26}$, S. Grohmann ${ }^{27}$, R. Größle ${ }^{19}$, R. Gumbsheimer ${ }^{19}$, V. Hannen ${ }^{5}$, S. Hansmann-Menzemer ${ }^{24}$, C. Hils ${ }^{4}$, B. Holzapfel ${ }^{27}$, J. Howlett ${ }^{9}$, G. Iaquaniello ${ }^{15}$, F. Jörg ${ }^{17}$, M. Keller ${ }^{24}$, J. Kellerer ${ }^{21}$, G. Khundzakishvili'18, B. Kilminster ${ }^{13}$, M. Kleifges ${ }^{12}$, T. K. Kleiner ${ }^{21}$, G. Koltmann ${ }^{10}$, A. Kopec $^{18}$, A. Kopmann ${ }^{12}$, L. M. Krauss ${ }^{28}$, F. Kuger ${ }^{14}$, L. LaCascio ${ }^{21}$, H. Landsman ${ }^{10}$, R. F. Lang ${ }^{18}$, S. Lindemann ${ }^{14}$, M. Lindner ${ }^{17}$, F. Lombardi ${ }^{6}$, J. A. M. Lopes ${ }^{6, d}$, A. Loya Villalpando ${ }^{7}$, Y. Ma ${ }^{29}$, C. Macolino ${ }^{15}$, J. Mahlstedt ${ }^{1}$, A. Manfredini13 ${ }^{13}$, T. Marrodán Undagoitia ${ }^{17}$, J. Masbou ${ }^{20}$, D. Masson ${ }^{14}$, E. Masson ${ }^{15}$, N. McFadden ${ }^{13}$, P. Meinhardt ${ }^{14}$, R. Meyer ${ }^{4}$, B. Milosevic ${ }^{8, e}$, S. Milutinovic ${ }^{8}$, A. Molinario ${ }^{25}$, C. M. B. Monteiro6 ${ }^{6}$ K. Morå9 E. Morteau $^{20}$, Y. Mosbacher ${ }^{10}$, M. Murra ${ }^{5}$, J. L. Newstead ${ }^{30}$, K. Ni ${ }^{29}$, U. G. Oberlack ${ }^{4}$, M. Obradovic ${ }^{8, e}$, K. Odgers ${ }^{16}$, I. Ostrovskiy $^{31}$, J. Palacio ${ }^{20}$, M. Pandurovic ${ }^{8}$, B. Pelssers ${ }^{1}$, R. Peres ${ }^{13}$, J. Pienaar ${ }^{26}$, M. Pierre ${ }^{20}$, V. Pizzella ${ }^{17}$, G. Plante ${ }^{9}$, J. Qi ${ }^{29}$, J. Qin ${ }^{18}$, D. Ramírez García ${ }^{14}$, S. E. Reichard ${ }^{13}$,a , N. Rupp ${ }^{17}$, P. Sanchez-Lucas ${ }^{13}$, J. M. F. dos Santos $^{6}$, G. Sartorelli ${ }^{2}$, D. Schulte ${ }^{5}$, H. C. Schultz-Coulon ${ }^{24}$, H. Schulze Eißing ${ }^{5}$, M. Schumann ${ }^{14}$, L. Scotto Lavina ${ }^{3}$, M. Selvi ${ }^{2}$, P. Shagin ${ }^{32}$, S. Sharma ${ }^{24}$, W. Shen ${ }^{24}$, M. Silva ${ }^{6}$, H. Simgen ${ }^{17}$, M. Steidl ${ }^{19}$, S. Stern ${ }^{21}$, D. Subotic ${ }^{8, e}$,

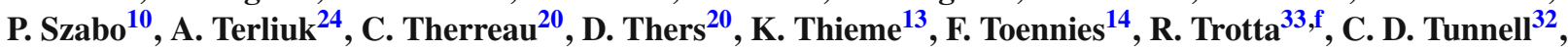
K. Valerius ${ }^{19}$, G. Volta $^{13}$, D. Vorkapic $^{8}$, M. Weber ${ }^{12}$, Y. Wei ${ }^{29}$, C. Weinheimer ${ }^{5}$, M. Weiss ${ }^{10}$, D. Wenz $^{14}$, C. Wittweg $^{5}$, J. Wolf ${ }^{21}$, S. Wuestling ${ }^{12}$, M. Wurm ${ }^{4}$, Y. Xing ${ }^{20}$, T. Zhu' ${ }^{9}$ Y. Zhu ${ }^{20}$, J. P. Zopounidis ${ }^{3}$, K. Zuber ${ }^{34}$

${ }^{1}$ Oskar Klein Centre, Department of Physics, Stockholm University, AlbaNova, 10691 Stockholm, SE, Sweden

2 Department of Physics and Astronomy, University of Bologna and INFN-Bologna, 40126 Bologna, Italy

${ }^{3}$ LPNHE, Université Pierre et Marie Curie, Université Paris Diderot, CNRS/IN2P3, 75252 Paris, France

${ }^{4}$ Institut für Physik \& Exzellenzcluster PRISMA ${ }^{+}$, Johannes Gutenberg-Universität Mainz, 55099 Mainz, Germany

${ }^{5}$ Institut für Kernphysik, Westfälische Wilhelms-Universität Münster, 48149 Münster, Germany

${ }^{6}$ LIBPhys, Department of Physics, University of Coimbra, 3004-516 Coimbra, Portugal

${ }^{7}$ Nikhef and the University of Amsterdam, Science Park, 1098XG Amsterdam, The Netherlands

${ }^{8}$ Vinca Institute of Nuclear Science, University of Belgrade, Mihajla Petrovica Alasa 12-14, Belgrade, Serbia

${ }^{9}$ Physics Department, Columbia University, New York, NY 10027, USA

${ }^{10}$ Department of Particle Physics and Astrophysics, Weizmann Institute of Science, 7610001 Rehovot, Israel

${ }^{11}$ New York University Abu Dhabi, Abu Dhabi, United Arab Emirates

12 Institute for Data Processing and Electronics (IPE), Karlsruhe Institute of Technology (KIT), 76344 Eggenstein-Leopoldshafen, Germany

13 Physik-Institut, University of Zurich, 8057 Zurich, Switzerland

${ }^{14}$ Physikalisches Institut, Universität Freiburg, 79104 Freiburg, Germany

15 Université Paris-Saclay, CNRS/IN2P3, IJCLab, 91405 Orsay, France

${ }^{16}$ Department of Physics, Applied Physics and Astronomy, Rensselaer Polytechnic Institute, Troy, NY 12180, USA

${ }^{17}$ Max-Planck-Institut für Kernphysik, 69117 Heidelberg, Germany

18 Department of Physics and Astronomy, Purdue University, West Lafayette, IN 47907, USA

${ }^{19}$ Institute for Astroparticle Physics (IAP), Karlsruhe Institute of Technology (KIT), 76344 Eggenstein-Leopoldshafen, Germany

20 SUBATECH, IMT Atlantique, CNRS/IN2P3, Université de Nantes, 44307 Nantes, France

${ }^{21}$ Institute of Experimental Particle Physics (ETP), Karlsruhe Institute of Technology (KIT), 76344 Eggenstein-Leopoldshafen, Germany

${ }^{22}$ Department of Physics and Chemistry, University of L'Aquila, 67100 L'Aquila, Italy

${ }^{23}$ INFN-Laboratori Nazionali del Gran Sasso and Gran Sasso Science Institute, 67100 L'Aquila, Italy

${ }^{24}$ Physikalisches Institut, Ruprecht-Karls-Universität Heidelberg, Heidelberg, Germany

25 INFN-Laboratori Nazionali del Gran Sasso and Gran Sasso Science Institute, 67100 L'Aquila, Italy

${ }^{26}$ Department of Physics and Kavli Institute for Cosmological Physics, University of Chicago, Chicago, IL 60637, USA 
${ }^{27}$ Institute for Technical Physics (ITEP), Karlsruhe Institute of Technology (KIT), 76344 Eggenstein-Leopoldshafen, Germany

28 The Origins Project Foundation, Phoenix, AZ 85020, USA

29 Department of Physics, University of California, San Diego, CA 92093, USA

${ }^{30}$ School of Physics, The University of Melbourne, Melbourne, VIC 3010, Australia

${ }^{31}$ Department of Physics, University of Alabama, Tuscaloosa, AL 35487, USA

32 Department of Physics and Astronomy, Rice University, Houston, TX 77005, USA

${ }_{33}$ Department of Physics, Imperial Centre for Inference and Cosmology, Imperial College London, London SW7 2AZ, UK

${ }^{34}$ Institute for Nuclear and Particle Physics, TU Dresden, 01069 Dresden, Germany

Received: 5 June 2020 / Accepted: 28 October 2020 / Published online: 8 December 2020

(C) The Author(s) 2020

\begin{abstract}
We detail the sensitivity of the proposed liquid xenon DARWIN observatory to solar neutrinos via elastic electron scattering. We find that DARWIN will have the potential to measure the fluxes of five solar neutrino components: $p p,{ }^{7} \mathrm{Be},{ }^{13} \mathrm{~N},{ }^{15} \mathrm{O}$ and pep. The precision of the ${ }^{13} \mathrm{~N},{ }^{15} \mathrm{O}$ and pep components is hindered by the doublebeta decay of ${ }^{136} \mathrm{Xe}$ and, thus, would benefit from a depleted target. A high-statistics observation of $p p$ neutrinos would allow us to infer the values of the electroweak mixing angle, $\sin ^{2} \theta_{w}$, and the electron-type neutrino survival probability, $P_{e e}$, in the electron recoil energy region from a few $\mathrm{keV}$ up to $200 \mathrm{keV}$ for the first time, with relative precision of $5 \%$ and $4 \%$, respectively, with 10 live years of data and a 30 tonne fiducial volume. An observation of $p p$ and ${ }^{7} \mathrm{Be}$ neutrinos would constrain the neutrino-inferred solar luminosity down to $0.2 \%$. A combination of all flux measurements would distinguish between the high- (GS98) and low-metallicity (AGS09) solar models with $2.1-2.5 \sigma$ significance, independent of external measurements from other experiments or a measurement of ${ }^{8} \mathrm{~B}$ neutrinos through coherent elastic neutrino-nucleus scattering in DARWIN. Finally, we demonstrate that with a depleted target DARWIN may be sensitive to the neutrino capture process of ${ }^{131} \mathrm{Xe}$.
\end{abstract}

\section{Introduction}

Current and future liquid xenon (LXe) direct detection dark matter experiments, such as XENONnT [1], LZ [2], and DARWIN [3], will exhibit sensitivity to neutrinos at the $\sim \mathrm{MeV}$ scale. Typically, neutrinos have been regarded as backgrounds in the search for dark matter (DM) [4,5];

\footnotetext{
a e-mail: shayne@physik.uzh.ch (Corresponding author)

b e-mail: darwin@lngs.infn.it

c Also at University of Banja Luka, Bosnia and Herzegovina

d Also at Institute for Subatomic Physics, Utrecht University, Utrecht, The Netherlands

e Wallenberg Academy Fellow

${ }^{\mathrm{f}}$ Also at Coimbra Polytechnic, ISEC, Coimbra, Portugal

g Also at Faculty of Mathematics

h Also at SISSA, Data Science Excellence Department, Trieste, Italy
}

but, as signals, they present opportunities to characterize their sources and pursue physics beyond the standard model (SM) [6-12]. While DM remains the primary objective, detectors with multi-tonne $(\mathrm{t})$ xenon targets will seek neutrino signals without the need for additional investment.

Solar neutrinos, in particular, are observable in dark matter detectors through two types of interactions: elastic electron scattering (ES) and coherent elastic neutrino-nucleus scattering (CEvNS) $[13,14]$. In the SM, ES is mediated by both charged and neutral currents. The former is only possible for $v_{e}$, which creates nearly an order of magnitude of difference between the interaction rates of $v_{e}$ and $v_{\mu, \tau}$. In total, the cross section for ES is $\sim 10^{-43} \mathrm{~cm}^{2}$. On the other hand, CEvNS is mediated only by neutral current, with a cross section of $\sim 10^{-39} \mathrm{~cm}^{2}$ that is strongly determined by the target's neutron number. With their different sensitivities to the components of the solar neutrino flux, these two channels provide complementarity over a wide range of neutrino energies.

Dedicated solar neutrino experiments have made numerous observations of ES with water, heavy water, and liquid scintillator targets. Borexino independently measured the fluxes of the lower-energy $p p$ [15], ${ }^{7} \mathrm{Be}$ [16], and pep [17] components. Subsequently, Borexino presented the first results from simultaneous spectroscopy of these three components above $0.19 \mathrm{MeV}$, yielding the most precise measurements to date $[18,19]$. Most recently, Borexino reported the first direct observation of carbon, nitrogen and oxygen (CNO) neutrinos at $5 \sigma$ [20]. Five experiments, Borexino [21,22], Super-K [23], KamLAND [24], SNO [25], and SNO+ [26], have measured the higher-energy ${ }^{8} \mathrm{~B}$ flux. COHERENT made the first observation of CEvNS [27], but astrophysical neutrinos have yet to be detected in this way.

After decades of investigation, important questions about the Sun persist. From an astrophysical perspective, the most salient issue lies in the solar abundance problem. The more recent low-metallicity (low-Z) AGSS09 SSM [28,29] would seem to better represent the photosphere than its predecessor, the high-metallicity (high-Z) GS98 SSM [28,30]. However, a comparison of individual flux measurements with theoretical predictions tends to favor the high-Z SSM, contradicting the common assumption that abundances in the radiative enve- 
lope are the same as those in the photosphere. This preference is further supported by helioseismic data that have long since disfavored a low-Z model [31]. As carbon, nitrogen and oxygen constitute the majority of heavy elements in the Sun, their neutrino fluxes are the most sensitive to metallicity. A combined analysis of available measurements remains inconclusive, but a relative uncertainty of $\sim 15 \%$ on a combined CNO flux measurement would begin to favor one model over another [19,32,33]. While less sensitive to metallicity than CNO neutrinos, an improved measurement of the ${ }^{8} \mathrm{~B}$ flux would also help to distinguish them further.

Measurements of electroweak and oscillation parameters play an important role in the understanding of the SM and the search for new physics $[34,35]$. Non-standard neutrino interactions (NSI) might modify the large mixing angle (MSWLMA) solution to the solar neutrino problem. Solar neutrinos serve as one probe to observe or set bounds on NSI. Two of these parameters, the electroweak mixing angle $\left(\sin ^{2} \theta_{w}\right)$ and the $v_{e}$ survival probability $\left(P_{e e}\right)$, which is the probability that a $v_{e}$ is detected on Earth as a $v_{e}$, may be measured with the ES process. The survival probability is determined by the elements of the unitary lepton mixing matrix. Only the higher energy solar neutrinos, ${ }^{8} \mathrm{~B}$ and hep, experience significant matter oscillation (MSW) effects. On one hand, atomic parity violation in cesium at $2.4 \mathrm{MeV}$ yields the lowest energy at which $\sin ^{2} \theta_{w}$ has been measured [36]. On the other, Borexino has provided the lowest-energy measurement of the $v_{e}$ survival probability extracted from the tail of the protonproton distribution $(>0.19 \mathrm{MeV})$ [19]. No experiment, thus far, has been able to access energies below these respective thresholds. DARWIN will measure $\sin ^{2} \theta_{w}$ and $P_{e e}$ for the first time in the (recoil) energy region $[1,200] \mathrm{keV}$.

In this manuscript, the authors highlight the efficacy with which DARWIN will shed light on solar and neutrino physics through elastic electron scattering. Details about the sensitivity to each component of the solar neutrino flux are provided first. Then, the precision with which DARWIN would reconstruct $\sin ^{2} \theta_{w}$ and $P_{e e}$ in the low energy range $[1,200] \mathrm{keV}$ is illustrated. Lastly, the extent to which a combined analysis of neutrino flux measurements would resolve the solar abundance problem is demonstrated.

\section{The DARWIN experiment}

The DARWIN observatory, which may begin operation as early as 2026, is a next-generation dark matter experiment that will operate with $50 \mathrm{t}$ ( $40 \mathrm{t}$ active) of xenon in a cylindrical, dual-phase time projection chamber (TPC) that is $2.6 \mathrm{~m}$ in both height and diameter [3]. The TPC will be placed underground in a double-walled cryostat vessel shielded by water Cherenkov and neutron vetoes that enable one to observe cosmogenic muons and their progeny. The TPC will be equipped to read out both light and charge signals. The location of the DARWIN observatory has not yet been determined. Discussions of background presume the overburden at the Laboratori Nazionali del Gran Sasso (3500 m water equivalent).

A charged particle that interacts in liquid xenon produces photons (scintillation) and electrons (ionization). The TPC promptly detects these photons as a primary "S1" scintillation signal with photosensors (PMTs, SiPMs, or an alternative) instrumented in arrays at the top and bottom of the target region. An electric field, $\mathscr{O}(0.1) \mathrm{kV} / \mathrm{cm}$, applied in the liquid volume drifts the electrons upward, and then a second field, $\mathscr{O}(1) \mathrm{kV} / \mathrm{cm}$, extracts them into the gas phase, where electroluminescence generates an amplified "S2" scintillation signal. The radial position of an interaction is reconstructed with the S2 light pattern in the top array, while its depth is inferred from the time delay between $\mathrm{S} 1$ and $\mathrm{S} 2$, up to $\mathscr{O}(1 \mathrm{~ms})$. Together, $\mathrm{S} 1$ and $\mathrm{S} 2$ reconstruct the energy of the event with excellent resolution. The charge-to-light ratio $\mathrm{S} 2 / \mathrm{S} 1$ discriminates between scatters off electrons and those off nuclei [37]. The combination of position, energy and discrimination allows for strong event selections to mitigate sources of background.

The most troublesome background for a solar neutrino search (ES) arises from the ${ }^{222} \mathrm{Rn}$ emanated by detector components. More precisely, the ${ }^{214} \mathrm{~Pb}$ daughter decays directly to ${ }^{214} \mathrm{Bi}$ with a branching ratio of $11 \%$, emitting a lone $\beta$ with an energy up to $Q=1.02 \mathrm{MeV}$ [38]. Otherwise, ${ }^{214} \mathrm{~Pb}$ decays to an excited state of ${ }^{214} \mathrm{Bi}$ that emits a $\gamma$ coincident with the $\beta$ to create a sharp rise above the lone- $\beta$ continuum. There are several excited states that contribute, starting at $0.274 \mathrm{MeV}$, as illustrated in Fig. 1. With a long halflife (3.8 days), ${ }^{222} \mathrm{Rn}$ distributes itself homogeneously in the LXe volume, such that it is not reduced with the deliberate selection of an inner volume, known as fiducialization. As radon mitigation is vital for the dark matter search, efforts are already underway to achieve a lower ${ }^{222} \mathrm{Rn}$ concentration. The detector materials in DARWIN will be screened for low radon emanation through a dedicated radioassay program, as in XENON1T/nT [39], to minimize the number of events in the low-energy region of interest. Gamma-ray spectroscopy and inductively-couple plasma mass spectrometry will provide systematic measurements of a wide variety of raw materials. The known activities of impurities will allow for the careful selection of these materials and the deliberate placement of fabricated components during construction. Furthermore, DARWIN is assumed to have a cryogenic radon distillation column integrated into the (gas) purification loop in order to counteract the continuous generation of ${ }^{222} \mathrm{Rn}$ from trace amounts of ${ }^{226} \mathrm{Ra}$. Radon accumulates in a reboiler at the bottom of the distillation column and remains there until disintegration. Meanwhile, the xenon is purified and extracted to the top, where it is reintroduced into the system. Cryogenic distillation has been successfully applied 
in XENON100 [40] and XENON1T [41]. In the latter case, a reduction of $\sim 20 \%$ was achieved. For this study, a target ${ }^{222} \mathrm{Rn}$ activity of $0.1 \mu \mathrm{Bq} / \mathrm{kg}$ is assumed.

A second background comes from intrinsic ${ }^{85} \mathrm{Kr}$, a $\beta$ emitter $\left(Q=0.687 \mathrm{MeV} ; \mathrm{T}_{1 / 2}=10.8\right.$ years $)$ that remains in the xenon volume after extraction from the atmosphere. As with ${ }^{222} \mathrm{Rn},{ }^{85} \mathrm{Kr}$ homogeneously distributes itself in the LXe volume. A krypton distillation column, designed to separate krypton and xenon through their different vapor pressures, may be deployed prior to a science run in order to reduce the krypton concentration to the desired level. The higher volatility of krypton in a static LXe reservoir leads to krypton enrichment in a gaseous xenon phase above. A portion of this enriched gas is removed at the top of the distillation column, and the purified xenon is extracted through the bottom. A series of distillation stages is needed to reach a separation factor greater than $10^{5}$. XENON1T has already demonstrated a concentration ${ }^{\text {nat }} \mathrm{Kr} / \mathrm{Xe}<360 \mathrm{ppq}$ [42]. Krypton may be further reduced at any time via online distillation, as applied in XENON1T $[41,43]$. A concentration of 2 ppq is assumed in this study, but - even at its current level - it has a negligible effect on measurements of flux, the electroweak mixing angle, and the electron-type survival probability. Only an observation of neutrino capture would require a $\mathscr{O}(\mathrm{ppq})$ concentration.

Long-lived radionuclides in detector materials constitute a third class of background events. The decay chains of ${ }^{238} \mathrm{U}$, ${ }^{232} \mathrm{Th}$ and ${ }^{235} \mathrm{U}$ generate various $\alpha$ and $\beta$ particles as well as $\gamma$ rays. The main contributors of $\gamma$ rays from these three chains are ${ }^{214} \mathrm{Bi}(2.45 \mathrm{MeV})$ and ${ }^{208} \mathrm{Tl}(2.61 \mathrm{MeV})$, including the background induced by decays in the non-instrumented xenon volume around the TPC. Additional $\gamma$ rays are emitted in the decays of ${ }^{137} \mathrm{Cs}(0.662 \mathrm{MeV}),{ }^{40} \mathrm{~K}(1.46 \mathrm{MeV})$, and the daughters of ${ }^{60} \mathrm{Co}(1.17$ and $1.33 \mathrm{MeV})$ and ${ }^{44} \mathrm{Ti}(2.66 \mathrm{MeV})$. The $\alpha$ and $\beta$ particles do not travel far and thus are eliminated with fiducialization. However, the $\gamma$ rays, with attenuation lengths up to $\sim 6 \mathrm{~cm}$ at $1 \mathrm{MeV}$, penetrate the innermost region, where they experience photoabsorption or Compton scattering. The more notable contributors have historically been the stainless steel cryostat and photosensors [39]. A materials background component derived from the DARWIN simulation in [44], which considers a more radiopure titanium cryostat, is included. The long-lived $\left(\mathrm{T}_{1 / 2}=59.1\right.$ years $)$, cosmogenically activated ${ }^{44} \mathrm{Ti}$ contributes to the materials background through its daughter ${ }^{44} \mathrm{Sc}$, which subsequently decays $\left(\mathrm{T}_{1 / 2}=3.8 \mathrm{~h}\right)$ with the emission of a $2.66 \mathrm{MeV}$ gamma. The simulated materials spectrum is adapted to this study by incorporating position-dependent multiscatter resolution, 3-15 mm, and selecting events within a $30 \mathrm{t}$ superellipsoidal fiducial volume that minimizes the contribution of these Compton scatters below recoil energies of $\sim 200 \mathrm{keV}$. With 10 live years of data, DARWIN would accrue 300 tonne- years (ty) of exposure, compared to the 200 ty goal for the dark matter search.

Finally, unstable xenon isotopes pose a potential background in the search for ES of solar neutrinos. The isotope ${ }^{136} \mathrm{Xe}$, which occurs naturally with an abundance of $8.9 \%$, undergoes double-beta decay $\left(Q=2.46 \mathrm{MeV} ; \mathrm{T}_{1 / 2}=\right.$ $2.17 \times 10^{21}$ years). The resultant spectrum circumscribes the entire signal region of interest. Furthermore, the cosmicmuon-induced neutron capture process of ${ }^{136} \mathrm{Xe}$ creates ${ }^{137} \mathrm{Xe}$, which then beta decays $\left(Q=4.16 \mathrm{MeV} ; \mathrm{T}_{1 / 2}=\right.$ $3.82 \mathrm{~min})$. The impact of ${ }^{137} \mathrm{Xe}$ proves to be negligible at the level of $10^{-3}$ per tonne-year per $\mathrm{keV}$, three orders of magnitude lower than ${ }^{136} \mathrm{Xe}$ double-beta decay [44]. These ${ }^{136}$ Xe background contributions are removable through isotopic depletion. The neutrinoless double-beta decay experiment EXO-200 [45], which uses a LXe volume enriched in ${ }^{136} \mathrm{Xe}$ to $80.6 \%$, has already demonstrated the possibility to alter the isotopic composition through ultracentrifugation [46]. Depletion, however, would diminish the prospects for a neutrinoless double-beta decay search with ${ }^{136} \mathrm{Xe}$ in DARWIN [44]. Lastly, ${ }^{124} \mathrm{Xe}$ decays via double electron capture $\left(\mathrm{T}_{1 / 2}=1.4 \times 10^{22}\right.$ years $)[47,48]$, as first observed in XENON1T [49]. The subsequent cascade of Auger electrons and X-rays is observed as a single peak at $64.3(36.7 ; 9.8) \mathrm{keV}$ with a branching ratio of $0.75(0.23 ; 0.017)$, following the fast atomic process and their sub-millimeter spread in liquid xenon. With an abundance of $0.1 \%$, one expects a total of 228 double electron capture events per tonne-year.

\section{Solar neutrinos in DARWIN}

DARWIN will be optimized for the detection of low-energy nuclear recoils. This fact also implies that DARWIN will be well equipped to detect ES with high efficiency and excellent energy resolution. In the following, the expected event rates for the individual solar components are calculated.

The spectral fluxes of $p p,{ }^{13} \mathrm{~N}$, and ${ }^{15} \mathrm{O}$ neutrinos are represented with the $\beta$ form,

$$
\frac{d \Phi_{i}}{d E_{v}}=\Phi_{i} A\left(x_{i}-E_{v}\right)\left[\left(x_{i}-E_{v}\right)^{2}-m_{e}^{2}\right]^{\frac{1}{2}} E_{v}^{2}
$$

where $x_{i} \equiv Q_{i}+m_{e}, Q_{i}$ and $\Phi_{i}$ are the characteristic maximal energy and the flux scale of neutrino component $i$, respectively, $m_{e}$ is the electron mass, $A$ is the corresponding normalization factor, and $E_{v}$ is the energy of the emitted neutrino. In contrast, ${ }^{7} \mathrm{Be}$ and pep neutrinos are monoenergetic. The ${ }^{7} \mathrm{Be}$ neutrinos are emitted at $0.862 \mathrm{MeV}(0.384 \mathrm{MeV})$ with a branching ratio of $90 \%(10 \%)$, while the pep neutrinos have an energy of $1.44 \mathrm{MeV}$. The flux scales are taken from the high-metallicity solar model [50]. 


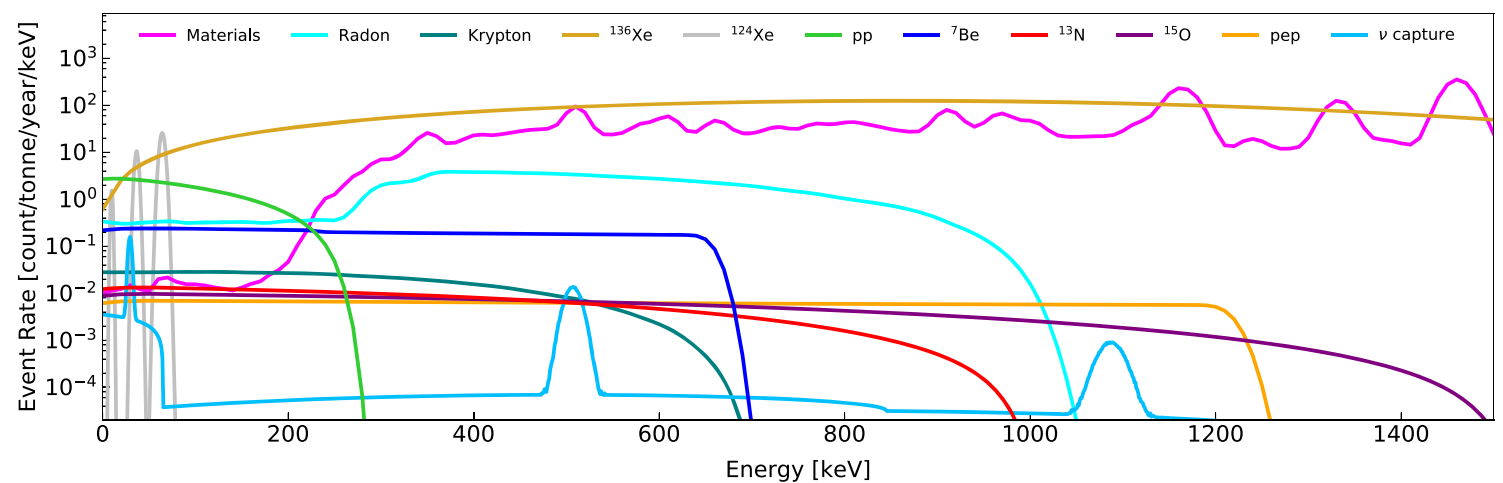

Fig. 1 The electron recoil spectra of five solar neutrino components, neutrino capture on ${ }^{131} \mathrm{Xe}$, and five backgrounds up to $1.5 \mathrm{MeV}$. The solar components follow from the high-Z SSM model. The materials and ${ }^{136} \mathrm{Xe}$ events in $[1.5,3] \mathrm{MeV}$ (not shown) are also used in the statistical analysis. The materials component is based on a selection of events in a $30 \mathrm{t}$ fiducial volume
These spectral fluxes are convolved with the differential cross section of elastic electron-neutrino scattering:

$\frac{d R_{i}}{d E_{r}}=N_{e} \sum_{j} \int P_{e j} \frac{d \Phi_{i}}{d E_{v}} \frac{d \sigma_{j}}{d E_{r}} d E_{v}$,

where $P_{e j}$ is the oscillation probability of lepton flavor $j$ to the electron neutrino, $N_{e}=2.48 \times 10^{29}$ is the number of target electrons per tonne of xenon, and $E_{r}$ is the energy of the induced recoil. The flux scales, maximum neutrino energies and survival probabilities are listed in Table 1. The values of survival probability follow the MSW-LMA solution at low energies in the vacuum-dominated regime [51]. The differential cross section is given by $[52,53]$

$$
\frac{d \sigma}{d E_{r}}=\frac{2 G_{F}^{2} m_{e}}{\pi}\left[g_{L}^{2}+g_{R}^{2}\left(1-\frac{E_{r}}{E_{v}}\right)^{2}-g_{L} g_{R} \frac{m_{e} E_{r}}{E_{v}^{2}}\right],
$$

with the coupling parameters $g_{L}=\sin ^{2} \theta_{w}-\frac{1}{2}$ and $g_{R}=$ $\sin ^{2} \theta_{w}$. For the $v_{e}, g_{L} \rightarrow g_{L}+1$ to account for its charged current interactions. A value of $\sin ^{2} \theta_{w}=0.2387$ [54] is assumed. In order to induce an electronic recoil, an incident neutrino must possess more energy than the binding energy of a given shell; and, when a recoil occurs, its energy is lowered accordingly. For this reason, xenon is not completely sensitive to neutrinos with the lowest energies. This effect is incorporated in the neutrino scattering rates with a step function defined by the series of discrete electron binding energies from $12 \mathrm{eV}$ to $35 \mathrm{keV}$. This ultimately leads to a suppression of a few percent in the $p p$ neutrino event rate and negligible reductions for the other solar neutrino components. The Gaussian energy resolution obtained in XENON1T [55], which remain unchanged with the step approximation, is also applied:

$$
\frac{\sigma\left(E_{r}\right)}{E_{r}}=\frac{0.3171}{\sqrt{E_{r}[\mathrm{keV}]}}+0.0015 .
$$

Table 1 The characteristic values of the flux scales [50], their relative uncertainties, the maximum neutrino energies, and the MSW-LMA $v_{e}$ survival probability [51] used in this study

\begin{tabular}{lllll}
\hline Component & $\Phi\left(\mathrm{cm}^{-2} \mathrm{~s}^{-1}\right)$ & $\sigma(\%)$ & $Q(\mathrm{keV})$ & $P_{e e}$ \\
\hline pp & $5.98 \times 10^{10}$ & 0.6 & 420 & 0.55 \\
${ }^{7} \mathrm{Be}$ & $4.93 \times 10^{9}$ & 6 & 862,384 & 0.52 \\
${ }^{13} \mathrm{~N}$ & $2.78 \times 10^{8}$ & 15 & 1200 & 0.52 \\
${ }^{15} \mathrm{O}$ & $2.05 \times 10^{8}$ & 18 & 1732 & 0.50 \\
pep & $1.44 \times 10^{8}$ & 1 & 1442 & 0.50 \\
\hline
\end{tabular}

The $p p$ neutrinos constitute the most prominent component due to the low energy threshold achievable in LXe TPCs. Here, a threshold of $1 \mathrm{keV}$ is assumed, yielding an integrated rate of 365 events per tonne-year. This high rate presents an opportunity to probe $\sin ^{2} \theta_{w}$ for the first time below $\sim 200 \mathrm{keV}$, to improve upon the precision of existing measurements of $P_{e e}$ at low energies, and to further constrain the neutrino-inferred measurement of solar luminosity.

The ${ }^{7} \mathrm{Be}$ neutrinos comprise the second most prominent component. The larger branch contributes 133 events per tonne-year, while the smaller one contributes 7.6 events. The ${ }^{7} \mathrm{Be}$ flux is more sensitive to solar metallicity and, as such, it may be combined with a high-precision measurement of the $p p$ flux to make an initial assessment of different metallicity models.

The third most prominent components are those of ${ }^{13} \mathrm{~N}$, ${ }^{15} \mathrm{O}$, and pep, which induce 6.5, 7.1 and 7.6 events per tonneyear, respectively. Despite having the lowest rate, ${ }^{13} \mathrm{~N}$ events fall within a narrower energy range than either ${ }^{15} \mathrm{O}$ or $p e p$, such that the ${ }^{13} \mathrm{~N}$ spectrum rises above both below $\sim 0.4 \mathrm{MeV}$. Consequently, it is possible for DARWIN to make a statistically significant observation of CNO neutrinos by exploiting higher statistics at lower energies. As the most sensitive to metallicity, being $30 \%$ higher in the high- $\mathrm{Z}$ scenario, mea- 
surements of the ${ }^{13} \mathrm{~N}$ and ${ }^{15} \mathrm{O}$ fluxes would greatly enhance the capability to distinguish between solar models. The rates of ${ }^{17} \mathrm{~F},{ }^{8} \mathrm{~B}$ and $h e p$ neutrinos are negligible.

Finally, the possibility of neutrino capture on ${ }^{131} \mathrm{Xe}(Q=$ $0.355 \mathrm{MeV}$ ) is considered. This isotope is the only one with a sufficiently low Q-value to exhibit sensitivity to solar neutrinos through this type of charged-current interaction. The expected observable signature consists of two signals: a prompt electron and a combination of X-rays and Auger electrons that are emitted together in the subsequent electron capture (EC) decay of ${ }^{131} \mathrm{Cs}^{+}\left(\mathrm{T}_{1 / 2}=9.69\right.$ days $)$. The prompt electrons would create a spectrum that mirrors those of the spectral neutrino fluxes shifted to lower energies by the $Q$-value of this reaction $\left(E_{e}=E_{v}-Q\right)$. The EC decay would appear as a Gaussian peak at $0.030 \mathrm{MeV}$. The long half-life of the EC process precludes delayed coincidence of these two signatures. The contribution of each solar component (including ${ }^{8} \mathrm{~B}$ ) follows from [56]. There are three distinct peaks visible in Fig. 1. The two higher energy peaks come from capturing the monoenergetic ${ }^{7} \mathrm{Be}$ and $p e p$ neutrinos; while the feature below $50 \mathrm{keV}$ is a combination of $\mathrm{EC}$, the lower branch of ${ }^{7} \mathrm{Be}$, and the tail of the $p p$ spectrum. In contrast to $p p$ neutrinos, the EC peak of the neutrino capture process provides a probe of the electron-type survival probability that depends on neutrinos in both the vaccuum- and matter-dominated regimes. As the five neutrino flux components considered here can be measured more precisely than the neutrino capture process, one may use the EC peak to infer the survival probability at high neutrino energies, as well, with an uncertainty comparable to current measurements. Accounting for both the survival probability and the ${ }^{131} \mathrm{Xe}$ abundance of $21.2 \%$, one expects 1.23 neutrino capture events per tonne-year with a natural xenon target.

\section{Flux and luminosity}

Having defined the signal and background models, an assessment of DARWIN's sensitivity to each of the neutrino components is made. A multivariate spectral fit of all $11 \mathrm{com}-$ ponents up to $3 \mathrm{MeV}$ is employed. Each signal and background component is represented by a single scale parameter in the fit. These scale parameters define the set of maximum likelihood estimators for the neutrino flux components, $\mathbf{f}=\left\{f_{p p}, f_{B e}, f_{N}, f_{O}, f_{p e p}\right\}$, and the neutrino capture rate, $f_{\text {cap }}$, given the electroweak and oscillation parameters, $\boldsymbol{\theta}=\left\{\sin ^{2} \theta_{w}, P_{e e}\right\}$ :

$P\left(n_{j} \mid \mu_{j}\left(\mathbf{f}, f_{\text {cap }}\right)\right)=\mathscr{L}\left(\mathbf{f}, f_{\text {cap }}\right)=\prod_{j=1} \frac{\mu_{j}^{n_{j}}}{n_{j} !} e^{-\mu_{j}}$.
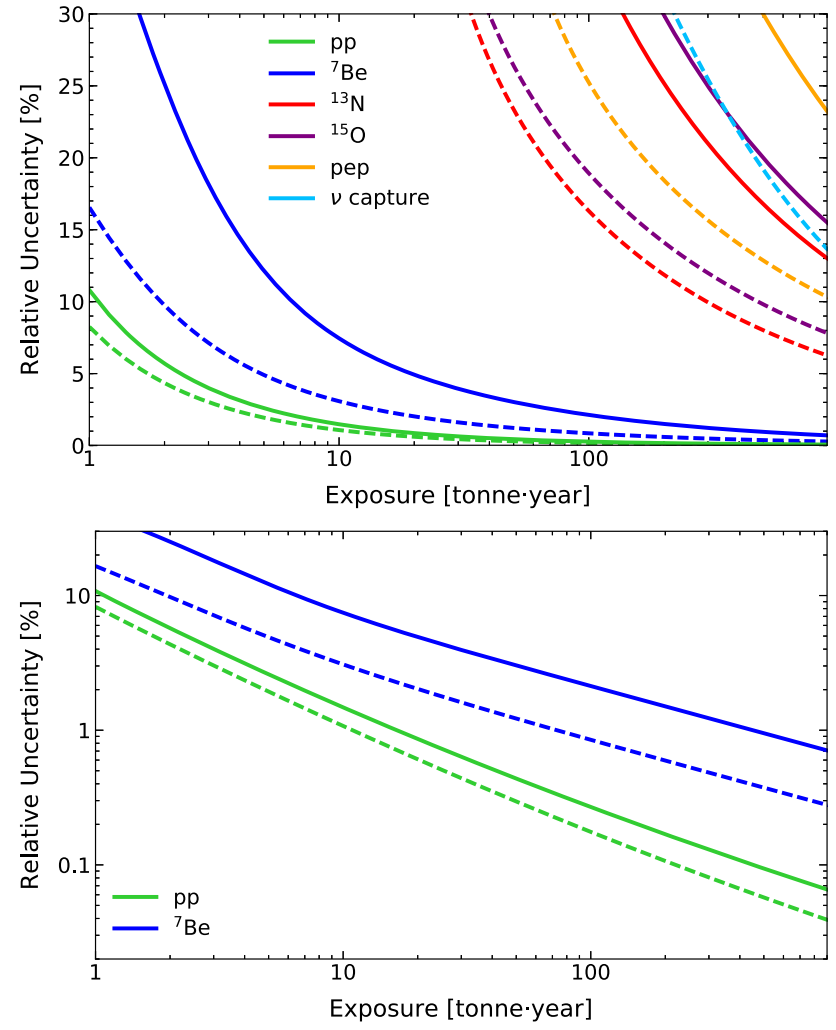

Fig. 2 The measured relative uncertainty of each solar neutrino component and neutrino capture as a function of exposure. The median fluxes of the high- $\mathrm{Z}$ model are assumed. Solid (dashed) curves correspond to a natural (depleted) target. A log scale of the $p p$ and ${ }^{7} \mathrm{Be}$ components is shown in the bottom panel for clarity

These two parameters, which are fixed to the values given in Sect. 3 for this low energy region, predict the average number of events in the $j$ th energy bin, $\mu_{j}$, while the observed number of events in that bin, $n_{j}$, is randomly sampled. The background-only region, $[1.5,3] \mathrm{MeV}$, is also used to constrain the uncertainties in the normalization of the materials and ${ }^{136} \mathrm{Xe}$ backgrounds at lower energies. The key advantage of the full spectral fit compared to a counting experiment in the low energy region of interest is that the constraints on the larger backgrounds, from ${ }^{136} \mathrm{Xe}$ and materials, may be improved from $\mathscr{O}(10) \%$ to $\mathscr{O}(0.1) \%$ or better. The scale of each signal and background component is left completely free throughout the fitting routine.

Toy experiments are run for each exposure in the range of interest, $[1,1000]$ ty, to ascertain the expected relative $1 \sigma$ uncertainties, $\sigma_{i}$, for each neutrino flux component. These uncertainties are shown in Fig. 2 normalized to their respective median high- $\mathrm{Z}$ flux values. The solid lines correspond to a natural target, while the dashed lines indicate a target depleted of ${ }^{136} \mathrm{Xe}$ by two orders of magnitude.

With 1 ty, DARWIN would quickly match the precision of the $p p$ flux (10\%) currently set by Borexino. A subpercent 

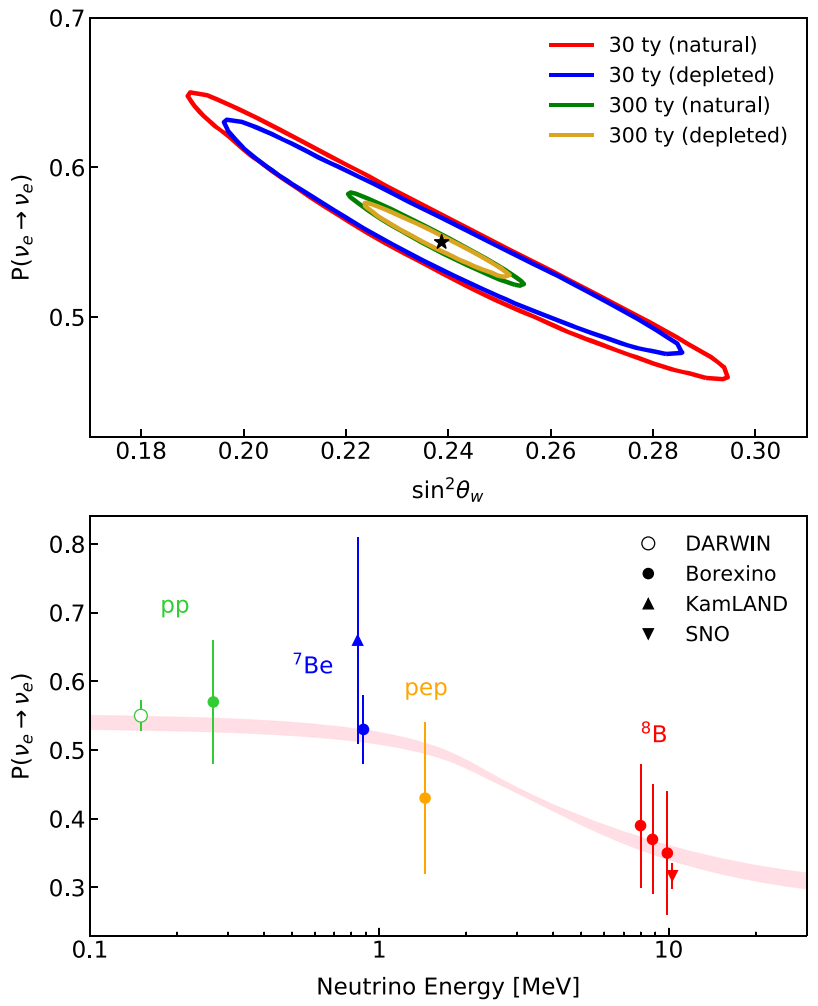

Fig. 3 (Top) The $68 \%$ confidence regions of $\sin ^{2} \theta_{w}$ and $P_{e e}$ for two exposures and the two target compositions. (bottom) The $v_{e}$ survival probability versus neutrino energy under the high-Z SSM. Dots represent the solar measurements of $p p$ (green), ${ }^{7} \mathrm{Be}$ (blue), pep (orange), and ${ }^{8} \mathrm{~B}$ (red) from Borexino $[19,22]$. The upward (downward) triangle shows a measurement of ${ }^{7} \mathrm{Be}\left({ }^{8} \mathrm{~B}\right)$ from KamLAND (SNO) $[24,25]$. The open point indicates DARWIN's projected enhancement of the precision of the $v_{e}$ survival probability to 0.02 below $420 \mathrm{keV}$ using $p p$ events. The pink band represents the $1 \sigma$ prediction of the MSW-LMA solution [51]

measurement would follow with 20 ty, ultimately reaching $0.15 \%$ at 300 ty. Similarly, DARWIN would match Borexino's ${ }^{7} \mathrm{Be}$ measurement (2.7\%) within 60 ty and then achieve $1 \%$ precision with 300 ty. The ${ }^{13} \mathrm{~N}$ and ${ }^{15} \mathrm{O}$ neutrinos would also be attainable as independent measurements. The former (latter) would require 100 ty (200 ty) to reach $3 \sigma$ detection with a natural xenon target. Finally, DARWIN could observe the pep component and neutrino capture process with 60 ty and 200 ty, respectively, using a necessarily depleted target.

The solar luminosity inferred from solar neutrino data, $L_{\odot, \nu} / L_{\odot}=1.04_{-0.08}^{+0.07}$, agrees with the measured (photoninferred) solar luminosity within $7 \%$ [32]. The $p p$ reaction contributes most strongly to the total energy generation in the Sun. Thus, high-precision measurements of the $p p$ and ${ }^{7}$ Be components, which respectively comprise $92 \%$ and $7.4 \%$ of the solar lumonisity, would reduce this uncertainty. With the precision levels shown here, DARWIN would achieve an uncertainty of $0.2 \%$ on the neutrino-inferred solar luminosity.

\section{Electroweak and oscillation parameters}

Following a precise measurement of the $p p$ component, one may infer the values of the electroweak mixing angle and the $v_{e}$ survival probability, as they directly affect the shape of its observed recoil spectrum. A likelihood function in which the electroweak and oscillation parameters are free to vary, while the flux scales (see Table 1) remain fixed, is adopted:

$P\left(n_{j} \mid \mu_{j}(\theta)\right)=\mathscr{L}(\theta)$.

The presence of ${ }^{7} \mathrm{Be}$ neutrinos only slightly worsens the sensitivity to these parameters, while the other neutrino components have a negligible effect. The uncertainty in the $p p$ flux contributes negligibly to the total uncertainty of $\sin ^{2} \theta_{w}$ and $P_{e e}$.

One finds the maximum likelihood estimators of $\sin ^{2} \theta_{w}$ and $P_{e e}$ in a series of toy experiments. From the resultant 2D distribution, the $68 \%$ confidence regions are determined, as shown in Fig. 3 (top), for four scenarios based on two exposures (30 and 300 ty) and two target compositions.

In the case of a natural target, DARWIN would reconstruct $\sin ^{2} \theta_{w}$ and $P_{e e}$ with uncertainties as small as 0.0122 $(5.1 \%)$ and $0.022(4.0 \%)$, respectively. Alternatively, with a depleted target, the uncertainties would shrink to 0.0099 $(4.2 \%)$ and $0.017(3.1 \%)$. A measurement of $\sin ^{2} \theta_{w}$ would be the first in this energy range, albeit with an uncertainty roughly five times higher than those at higher energies. A measurement of $P_{e e}$ would improve upon the existing one from Borexino by an order of magnitude. This projection is shown in Fig. 3 (bottom) with solar neutrino measurements from Borexino [19,22], KamLAND [24], and SNO [25].

\section{Solar abundance problem}

DARWIN may utilize a combination of neutrino flux measurements to probe the metallicity of the Sun. The sensitivity assessment of the flux components, $\mathbf{f}$, described in Sect. 4 is repeated with the same backgrounds. In this instance, however, the median flux values of the high- and low- $\mathrm{Z}$ models are allowed to vary according to their respective theoretical uncertainties. These uncertainties, $\sigma_{i}$, are then put into a multivariate (Gaussian) simulation characterized by a 5dimensional matrix $\Sigma=\left[\rho_{i j} \sigma_{i} \sigma_{j}\right]$ that accounts for all correlations of the flux components, $\rho_{i j}$. The correlation values are based on [50]. For each trial at a given exposure, the randomly sampled $\mathbf{f}$ is used to calculate a (squared) Mahalanobis distance $\delta^{2}=(\mathbf{f}-1)^{T} \cdot \Sigma^{-1} \cdot(\mathbf{f}-1)$ [57], which quantifies the deviation from the true (median) values. The simulation is repeated for both the high- and low-Z models. A p-value is calculated for each iteration of the high- $Z$ simulation by integrating the low-Z Mahalanobis distribution above the high- $Z$ 


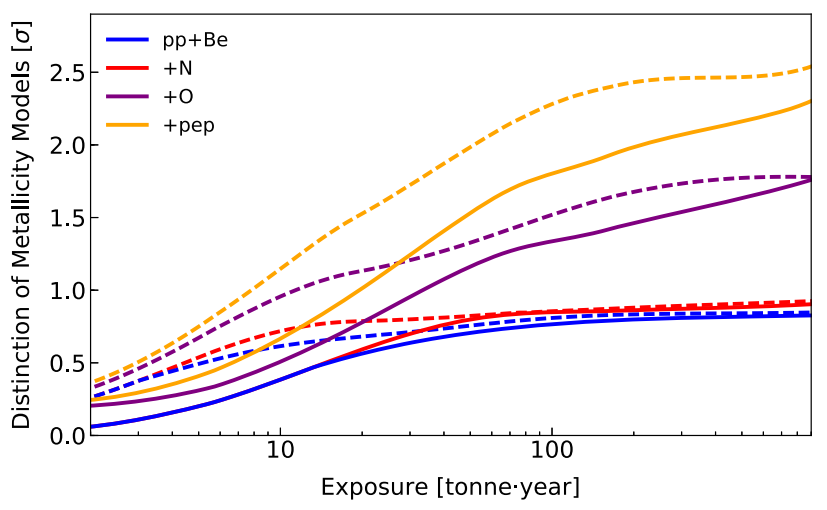

Fig. 4 The median significance with which the the high- and low-Z models may be distinguished is calculated for the first pair of flux measurements, $p p$ and ${ }^{7} \mathrm{Be}$, as a function of exposure. Additional cases add ${ }^{13} \mathrm{~N},{ }^{15} \mathrm{O}$ and pep sequentially. The solid (dashed) curves correspond to a natural (depleted) xenon target

Mahalanobis value. The median p-value is then taken from its final distribution.

Figure 4 displays the significance corresponding to these p-values as a function of exposure for the first pair of measurements, $p p$ and ${ }^{7} \mathrm{Be}$, and for each subsequent addition of the other components. The ${ }^{13} \mathrm{~N}$ component only modestly increases the exclusion potential due to the large uncertainties in both theory and experiment. The combination of both ${ }^{13} \mathrm{~N}$ and ${ }^{15} \mathrm{O}$, however, yields a significant gain above $\sim 10$ ty. The pep component enhances the exclusion to $2.1 \sigma(2.5 \sigma)$ with a natural (depleted) target at an exposure of 300 ty. The series of improvements in the distinction power with each new measurement does not necessarily reflect the intrinsic properties of the corresponding neutrino component or its correlation to solar metallicity. Rather, it represents DARWIN's specific ability to discern one model from another using up to five measurements simultaneously, at a given exposure.

DARWIN would remain limited by the ${ }^{136} \mathrm{Xe}$ background with a natural target, but with depletion it would distinguish between the high- and low-Z SSMs up to the theoretical uncertainties. The significance illustrated in Fig. 4 may be further improved either with a measurement of the solar ${ }^{8} \mathrm{~B}$ flux via CEvNS in DARWIN or with independent measurements from other experiments.

\section{Outlook}

The DARWIN observatory will feature sensitivity to five components of the solar flux via ES. A low energy threshold of $1 \mathrm{keV}$ allows DARWIN to observe the majority of $p p$ neutrinos, which have (mostly) eluded contemporary neutrino observatories. With 300 ty, DARWIN would be able to achieve $0.15 \%$ precision in the $p p$ flux measurement, approximately two orders of magnitude better than the current preci- sion from Borexino. DARWIN would improve upon existing measurements of the ${ }^{7} \mathrm{Be}$ flux by a factor of 3 . These measurements, in turn, would reduce the uncertainty on the neutrinoinferred solar luminosity to $0.2 \%$. The pep neutrinos may be observed with $3 \sigma$ significance within the lifetime of the experiment, depending on the target composition. And, with only three years of data, DARWIN would make independent observations of CNO neutrinos with $3 \sigma$ significance.

Precise measurements of these solar components further extend the physics reach of DARWIN. The high-statistics $p p$ events would provide the means to measure both $\sin ^{2} \theta_{w}$ and $P_{e e}$ in an energy region that is yet to be probed. The precision of $P_{e e}$, in particular, would be up to one order of magnitude better than the current lowest-energy measurement from Borexino. All obtained measurements and limits on the fluxes would together provide information to distinguish between the high- and low-Z SSMs. These capabilities are dependent on the target composition. Only with a target depleted of ${ }^{136} \mathrm{Xe}$ by approximately two orders of magnitude would DARWIN make such precise measurements via ES or exploit them to distinguish between solar models. DARWIN may further enhance its distinction power with a measurement of the ${ }^{8} \mathrm{~B}$ flux via CEvNS. The forecast for such a measurement is highly sensitive to the achieved energy threshold for nuclear recoils, and it is left for a future study. A powerful physics case exists for the pursuit of solar neutrinos in DARWIN, and it comes without the need for additional investment beyond the option of depletion.

Data published in this article are available in [58].

Acknowledgements This work was supported by the Swiss National Science Foundation under grants Nos. 200020-162501, and 200020175863, by the European Union's Horizon 2020 research and innovation programme under the Marie Sklodowska-Curie grant agreements Nos. 674896, 690575, and 691164, by the European Research Council (ERC) grant agreements No. 742789 (Xenoscope), and No. 724320 (ULTIMATE), by the Max-Planck-Gesellschaft, by the Deutsche Forschungsgemeinschaft (DFG) under GRK-2149, by the US National Science Foundation (NSF) grants No. 1719271, and No. 1940209, by the Portuguese FCT, by The Netherlands Organisation for Scientific Research (NWO), by the Ministry of Education, Science and Technological Development of the Republic of Serbia and by grant ST/N000838/1 from Science and Technology Facilities Council (UK).

Data Availability Statement This manuscript has associated data in a data repository. [Author's comment: The data generated in this study are available in the named repository (https://github.com/ Physik-Institut-UZH/DARWIN-Sensitivity-Studies/tree/master/solar _neutrinos_electron_scattering).]

Open Access This article is licensed under a Creative Commons Attribution 4.0 International License, which permits use, sharing, adaptation, distribution and reproduction in any medium or format, as long as you give appropriate credit to the original author(s) and the source, provide a link to the Creative Commons licence, and indicate if changes were made. The images or other third party material in this article are included in the article's Creative Commons licence, unless indicated otherwise in a credit line to the material. If material is not 
included in the article's Creative Commons licence and your intended use is not permitted by statutory regulation or exceeds the permitted use, you will need to obtain permission directly from the copyright holder. To view a copy of this licence, visit http://creativecomm ons.org/licenses/by/4.0/.

Funded by SCOAP ${ }^{3}$.

\section{References}

1. E. Aprile et al. (XENON Collaboration). arXiv:2007.08796

2. D.S. Akerib et al. (LUX-ZEPLIN Collaboration). arXiv: 1802.06039

3. J. Aalbers et al. (DARWIN Collaboration), JCAP 1611 , 017 (2016). https://doi.org/10.1088/1475-7516/2016/11/017. arXiv: 1606.07001

4. L.E. Strigari, New J. Phys. 11, 105011 (2009). https://doi.org/10. 1088/1367-2630/11/10/105011. arXiv:0903.3630

5. J. Billard, L. Strigari, E. Figueroa-Feliciano, Phys. Rev. D 89, 023524 (2014). https://doi.org/10.1103/PhysRevD.89.023524. arXiv: 1307.5458

6. R.F. Lang et al., Phys. Rev. D 94, 103009 (2016). https://doi.org/ 10.1103/PhysRevD.94.103009. arXiv:1606.09243

7. L. Baudis et al., JCAP 01, 044 (2014). https://doi.org/10.1088/ 1475-7516/2014/01/044. arXiv:1309.7024

8. J.L. Newstead, L.E. Strigari, R.F. Lang, Phys. Rev. D 99, 043006 (2019). https://doi.org/10.1103/PhysRevD.99.043006. arXiv:1807.07169

9. B. Dutta et al., Phys. Lett. B 773, 242-246 (2017). https://doi.org/ 10.1016/j.physletb.2017.08.031. arXiv:1705.00661

10. D.G. Cerdeño et al., JHEP 05, 118 (2016). https://doi.org/10.1007/ JHEP09(2016)048. arXiv:1604.01025 [Erratum: JHEP 09, 048 (2016)]

11. D. Aristizabal Sierra, N. Rojas, M.H.G. Tytgat, JHEP 03, 197 (2018). https://doi.org/10.1007/JHEP03(2018)197. arXiv: 1712.09667

12. M.C. Gonzalez-Garcia et al., JHEP 07, 019 (2018). https://doi.org/ 10.1007/JHEP07(2018)019. arXiv: 1803.03650

13. J. Billard, L.E. Strigari, E. Figueroa-Feliciano, Phys. Rev. D 91, 095023 (2015). https://doi.org/10.1103/PhysRevD.91.095023. arXiv: 1409.0050

14. D. Akimov et al. (COHERENT Collaboration), Science 357(6356), 1123 (2017). https://doi.org/10.1103/PhysRevLett.122.141301. arXiv:1708.01294

15. G. Bellini et al. (Borexino Collaboration), Nature 512, 383 (2014). https://doi.org/10.1038/nature13702

16. G. Bellini et al. (Borexino Collaboration), Phys. Rev. Lett. 107, 141302 (2011). https://doi.org/10.1103/PhysRevLett.107.141302. arXiv:1104.1816

17. G. Bellini et al. (Borexino Collaboration), Phys. Rev. Lett. 108, 051302 (2012). https://doi.org/10.1103/PhysRevLett.107.141302. arXiv: 1110.3230

18. M. Agostini et al. (Borexino Collaboration), Phys. Rev. D 100 , 082004 (2019). https://doi.org/10.1103/PhysRevD.100.082004. arXiv:1707.09279

19. M. Agostini et al. (Borexino Collaboration), Nature 562, 505 (2018). https://doi.org/10.1038/s41586-018-0624-y

20. M. Agostini et al. (Borexino Collaboration). arXiv:2006.15115

21. G. Bellini et al. (Borexino Collaboration), Phys. Rev. D 82, 033006 (2010). https://doi.org/10.1103/PhysRevD.82.033006. arXiv:0808.2868

22. M. Agostini et al. (Borexino Collaboration), Phys. Rev. D 101, 062001 (2020). https://doi.org/10.1103/PhysRevD.101.062001. arXiv:1709.00756
23. K. Abe et al. (Super-Kamiokande Collaboration), Phys. Rev. D 83, 052010 (2011). https://doi.org/10.1103/PhysRevD.83.052010. arXiv: 1010.0118

24. S. Abe et al. (KamLAND Collaboration), Phys. Rev. C 84, 035804 (2011). https://doi.org/10.1103/PhysRevC.84.035804. arXiv: 1106.0861

25. B. Aharmim et al. (SNO Collaboration), Phys. Rev. C 88, 025501 (2013). https://doi.org/10.1103/PhysRevC.88.025501. arXiv: 1109.0763

26. M. Anderson et al. (SNO+ Collaboration), Phys. Rev. D 99, 012012 (2018). https://doi.org/10.1103/PhysRevD.99.012012. arXiv: 1812.03355

27. D. Akimov et al. (COHERENT Collaboration), Science 357, 1123-1126 (2017). https://doi.org/10.1126/science.aao0990. arXiv: 1708.01294

28. A. Serenelli, Eur. Phys. J. A 52(4), 78 (2016). https://doi.org/10. 1140/epja/i2016-16078-1. arXiv:1601.07179

29. M. Asplund et al., Ann. Rev. Astron. Astrophys. 47, 481 (2009). https://doi.org/10.1146/annurev.astro.46.060407.145222. arXiv:0909.0948

30. N. Grevesse, A.J. Sauval, Space Sci. Rev. 85, 161 (1998). https:// doi.org/10.1023/A:1005161325181

31. A. Serenelli et al., Astrophys. J. 705, L123 (2009). https://doi.org/ 10.1088/0004-637X/705/2/L123. arXiv:0909.2668

32. J. Bergstrom et al., JHEP 03, 132 (2016). https://doi.org/10.1007/ JHEP03(2016)132. arXiv:1601.00972

33. M. Agostini et al. (Borexino Collaboration). arXiv:2005.12829

34. K.S. Kumar et al., Ann. Rev. Nucl. Part. Sci. 63, 237 (2013). https:// doi.org/10.1146/annurev-nucl-102212-170556. arXiv:1302.6263

35. M. Maltoni, A.Y. Smirnov, Eur. Phys. J. A 52, 87 (2016). https:// doi.org/10.1140/epja/i2016-16087-0. arXiv:1507.05287

36. H. Davoudiasl, H.-S. Lee, W.J. Marciano, Phys. Rev. D 92, 055005 (2015). https://doi.org/10.1103/PhysRevD.92.055005. arXiv: 1507.00352

37. V.N. Lebedenko et al., Phys. Rev. D 80, 052010 (2009). https://doi. org/10.1103/PhysRevD.80.052010. arXiv:0812.1150

38. Brookhaven National Laboratory, national nuclear data center (2015)

39. E. Aprile et al. (XENON Collaboration), Eur. Phys. J. C 77, 890 (2017). https://doi.org/10.1140/epjc/s10052-017-4757-1. arXiv: 1705.01828

40. E. Aprile et al. (XENON Collaboration), Eur. Phys. J. C 77, 358 (2017). https://doi.org/10.1140/epjc/s10052-017-4902-x. arXiv: 1702.06942

41. M. Murra, Intrinsic background reduction by cryogenic distillation for the XENON1T darkmatter experiment, Ph.D. thesis, WWU Münster (2019)

42. E. Aprile et al. (XENON Collaboration), Phys. Rev. D 99, 112009 (2019). https://doi.org/10.1103/PhysRevD.99.112009. arXiv: 1902.11297

43. E. Aprile et al. (XENON Collaboration), Eur. Phys. J. C 77, 275 (2017). https://doi.org/10.1140/epjc/s10052-017-5329-0. arXiv: 1612.04284

44. F. Agostini et al. (DARWIN Collaboration), Eur. Phys. J. C 80, 808 (2020). https://doi.org/10.1140/epjc/s10052-020-8196-z. arXiv:2003.13407

45. G. Anton et al. (EXO-200 Collaboration), Phys. Rev. Lett. 123, 161802 (2019). https://doi.org/10.1103/PhysRevLett.123.161802. arXiv: 1906.02723

46. M. Auger et al., (EXO-200 Collaboration), JINST 7, P05010 (2012). https://doi.org/10.1088/1748-0221/7/05/P05010. arXiv: 1202.2192

47. C. Wittweg et al., (2020). arXiv:2002.04239

48. M. Doi, T. Kotanit, Prog. Theor. Phys. 87, 5 (1992). https://doi.org/ $10.1143 / \mathrm{ptp} / 87.5 .1207$ 
49. E. Aprile et al. (XENON Collaboration), Nature 568, 7753 (2019). https://doi.org/10.1038/s41586-019-1124-4. arXiv:1904.11002

50. N. Vinyoles et al., Astrophys. J. 835, 202 (2017). https://doi.org/ 10.3847/1538-4357/835/2/202. arXiv:1611.09867

51. F. Capozzi et al., Phys. Rev. D 95, 096014 (2017). https://doi.org/ 10.1103/PhysRevD.95.096014. arXiv:1703.04471

52. W.J. Marciano, Z. Parsa, J. Phys. G29, 2629 (2003). https://doi. org/10.1088/0954-3899/29/11/013. arXiv:hep-ph/0403168

53. J.A. Formaggio, G.P. Zeller, Rev. Mod. Phys. 84, 1307 (2012). https://doi.org/10.1103/RevModPhys.84.1307. arXiv:1305.7513

54. J. Erler, M.J. Ramsey-Musolf, Phys. Rev. D 72, 073003 (2005). $\quad$ https://doi.org/10.1103/PhysRevD.72.073003. arXiv:hep-ph/0409169

55. E. Aprile et al. (XENON Collaboration), (2020).arXiv:2003.03825
56. ASh Georgadze et al., Astropart. Phys. 7, 173 (1997). https://doi. org/10.1016/S0927-6505(97)00017-0

57. P.C. Mahalanobis, Proc. Natl. Inst. Sci. India 2, 49 (1936)

58. Data Repository, Physik-Institut, University of Zurich. https:// github.com/Physik-Institut-UZH/DARWIN-Sensitivity-Studies/ tree/master/solar_neutrinos_electron_scattering 